\title{
Assemblability analysis of the modular fixturing systems with accurate grid pattern holes using Monte Carlo simulation
}

\author{
Tudor Păunescu ${ }^{1, *}$ \\ ${ }^{1}$ Transilvania University of Brasov, Department of Manufacturing Engineering, Mihai Viteazu No.5, \\ Braşov, Romania.
}

\begin{abstract}
This paper focuses on the assemblability of modular fixturing systems. Two types of stochastic models are proposed: for two holes-two pins $(2 \mathrm{H} 2 \mathrm{P})$ and for two holes-two pins-two holes $(2 \mathrm{H} 2 \mathrm{P} 2 \mathrm{H})$, both used by modular fixturing systems with multipurpose grid pattern holes. The probability of successful assemblies to engineering specifications is calculated using the Monte Carlo simulation. The weights of the assemblability criterion variables are analysed and several design recommendations are indicated.
\end{abstract}

\section{Introduction}

Modular fixtures (MF) is one of the most appropriate flexible fixturing techniques used by CIM systems. It is well known that MF is a workholding using standardized reusable components. After the fixture is built and the machining process is completed, the fixture can be disassembled and the elements can be used to build another fixture. MF fills the gap between the general-purpose and special-purpose fixtures.

A lot of academic and applications papers have been published in this area, much focus has been put on intelligent methods for computer aided fixture $[1,2]$.

The present paper focuses on modular fixturing systems (MFS) with grid pattern holes (MFSH) because offer several advantages over modular systems with $\mathrm{T}$ slots: more positional possibilities of the modules on the base plate, more security, repeated setups are easier, if grid pattern holes are damaged, these are repairable.

MFSHs are based on alternating dowel holes and tapped holes, or multipurpose holes. The second is very popular, because the coaxial arrangement of threaded and cylindrical locating surface allows positioning and fastening at the same time.

The MFSHs use two locating principles of modules: if the locating module has a symmetry axis one hole - one pin - one hole assembly is used; if the locating module has not a symmetry axis the assembly is based on two holes-two pins-two holes.

The pins are rigid bodies (position sleeves (fig. 1a), position screws (fig. 1c)) or elastic bodies (slotted position sleeves (fig. 1b), collets).

Is necessary to analyse the assemblability of the modules, as first step of optimizing tolerances.

\footnotetext{
* Corresponding author: t.paun@unitbv.ro
} 


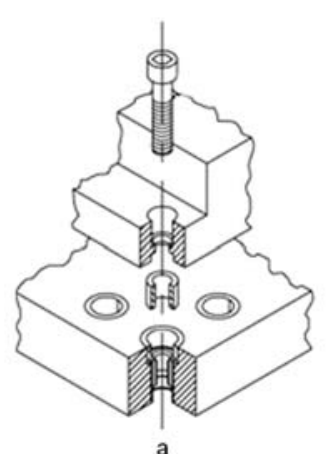

a

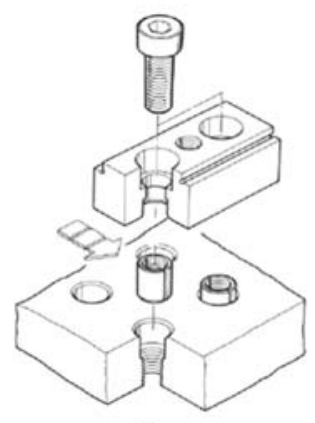

b

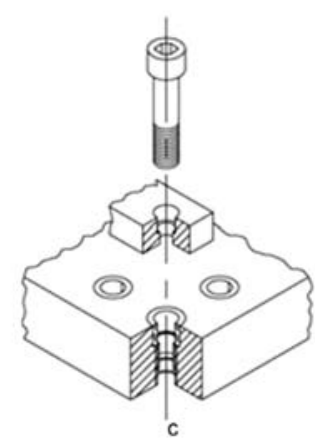

Fig. 1. Three positioning systems based on: rigid sleeve (a), slotted sleeve (b) and position screw (c) $[3-6]$.

Grid pattern of MFSH for medium-size workpieces are spaced 25, 40, $50 \pm 0.01 \mathrm{~mm}$, on every grid tooling plate or blocks. Usually, the holes and pins have 5-7 IT grades (Table 1).

Table 1. Tolerances of the MFSH.

\begin{tabular}{|c|l|c|c|c|c|}
\hline No. & \multicolumn{1}{|c|}{ MFSH } & $\begin{array}{c}\mathrm{D}_{1} \\
{[\mathrm{~mm}]}\end{array}$ & $\begin{array}{c}\mathrm{D}_{2} \\
{[\mathrm{~mm}]}\end{array}$ & $\begin{array}{c}\mathrm{D}_{3} \\
{[\mathrm{~mm}]}\end{array}$ & $\begin{array}{c}\text { pitch } \\
{[\mathrm{mm}]}\end{array}$ \\
\hline 1 & AMF M12 [4] & $\Phi 16 \mathrm{~F} 7$ & $\Phi 16_{0.00}^{0.011}$ & $\Phi 16 \mathrm{~F} 7$ & $40 \pm 0.01$ \\
\hline 2 & AMF M16 [4] & $\Phi 22 \mathrm{~F} 7$ & $\Phi 22_{0.002}^{0.015}$ & $\Phi 22 \mathrm{~F} 7$ & $50 \pm 0.01$ \\
\hline 3 & Halder M12 [5] & $\Phi 12 \mathrm{~F} 6$ & $\Phi 12 \mathrm{k} 5$ & $\Phi 12 \mathrm{~F} 6$ & $50 \pm 0.01$ \\
\hline 4 & Halder M16 [5] & $\Phi 16 \mathrm{~F} 6$ & $\Phi 16 \mathrm{k} 5$ & $\Phi 16 \mathrm{~F} 6$ & $50 \pm 0.01$ \\
\hline 5 & Kipp M8 [6] & $\Phi 12 \mathrm{H} 6$ & $\Phi 12 \mathrm{~h} 6$ & $\Phi 12 \mathrm{H} 6$ & $25 \pm 0.01$ \\
\hline 6 & Kipp M12,16 [6] & $\Phi 12,16 \mathrm{~F} 7$ & $\Phi 12,16 \mathrm{~g} 5$ & $\Phi 12,16 \mathrm{H} 7$ & $50 \pm 0.01$ \\
\hline 7 & Norelem M6 [7] & $\Phi 12 \mathrm{E} 6$ & $\Phi 12 \mathrm{n} 6$ & $\Phi 12 \mathrm{E} 6$ & $25 \pm 0.01$ \\
\hline 8 & Norelem M10 [7] & $\Phi 18 \mathrm{E} 6$ & $\Phi 18 \mathrm{n} 6$ & $\Phi 18 \mathrm{E} 6$ & $40 \pm 0.01$ \\
\hline
\end{tabular}

Notations: $\mathrm{D}_{\mathrm{i}}, \mathrm{i}=1-3$, centering diameter; 1-fixture base; 2-locating component (locating screw or sleeve); 3-modular mounted component.

\section{The assemblability problem of modular fixturing systems}

The usual solving methods for assemblability problem are: the worst case method (WCM) and statistical methods, mainly Monte Carlo simulations (MCS). Statistical tolerance design is accepted by industry, particularly in case of modular fixturing systems if a module fails to assemble it can be replaced with another interchangeable module. Because WCM assumes extreme conditions that rarely happen, the percent of components which cannot be assembled is zero. The MCS can generate a more reasonable results than WCM, because is possible to increase the tolerances for individual dimensions, in this way manufacturing costs could be reduced.

The assemblability problem calculates the probability of successfully parts assembling when the dimensions and tolerances of the parts, mating constraints, and an assembly sequence are given. In case of modular fixture, an assembly sequence is not required because each locating module has unique assembly scheme.

The main sources of errors are: geometric errors, elastic deformations due to fixturing and cutting forces, wear and thermic deformations. In this approach, geometrical models of positioning modules are considered. Also, suppose that the locating modules has a low ratio of height to length, the geometrical models are simplified to 2D. 


\subsection{Assemblability of $2 \mathrm{H} 2 \mathrm{P}$}

The 2H2P assembly is used by the Norelem MFSH [7] for two components: rest and seating ledge (fig. 2) and riser block plug-in for locating pin.
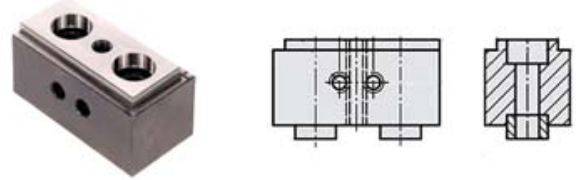

Fig. 2. Support-stop block with force fits between the sleeves and the holes of module [7].
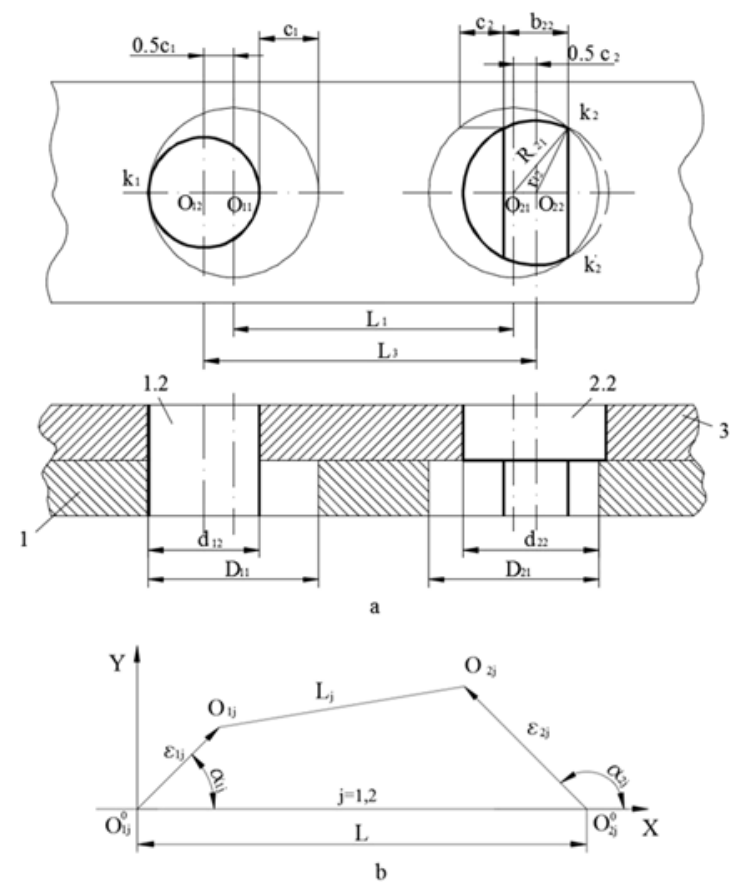

Fig. 3. a-2H2P in a limit assembly position; b-vectorial summation of centre distances.

\section{Notations}

Indexes for $\mathrm{D}_{\mathrm{ij}}, \mathrm{d}_{\mathrm{ij}}, \mathrm{UL}_{\mathrm{j}}, \mathrm{LL}_{\mathrm{j}}, \mathrm{ul}_{\mathrm{j}}, \mathrm{ll}_{\mathrm{j}}, \mathrm{c}_{\mathrm{j}}, \mathrm{L}_{\mathrm{i}}, \alpha_{\mathrm{ij}}, \varepsilon_{\mathrm{ij}}$ :

$\mathrm{i}=1$-3: 1-base module; 2 - rigid sleeve; 3 - mounted module;

$\mathrm{j}=1,2$ : 1 - for round sleeve; 2 - for diamond sleeve and corresponding hole;

$\mathrm{L}_{\mathrm{i}}, \mathrm{i}=1-3$ centre distance between axes;

$D_{i j}, d_{i j}, i=1-3, j=1,2$ diameters of holes and sleeves respectively;

$c_{j}, j=1,2$ clearances between the holes and sleeves;

$\mathrm{TPp}_{\mathrm{i}}, \mathrm{i}=1,3$ the positional tolerance of the centering hole;

$\mathrm{TP}_{22}$ locating diamond pin symmetry tolerance;

$\mathrm{T}_{22}$ tolerance of the diamond pin width;

$\mathrm{UL}_{\mathrm{j}}, \mathrm{LL}_{\mathrm{j}}, \mathrm{ul}_{\mathrm{j}}, \mathrm{ll}_{\mathrm{j}}, \mathrm{j}=1,2$ upper and lower limits for holes and pins respectively;

m-minimum, M-maximum.

Basic sizes of holes and sleeves/pins are equal, and the limits are equal respectively.

The same rule is applied for basic size of centre distance between the two holes. 
A lot of MFSH producers use the conventional tolerancing $( \pm 0.5 \mathrm{~T})$ of pitch. If the ISO position tolerance is used, is necessary to make following substitution: $\mathrm{TPp} \rightarrow 0.5 \mathrm{~T}$.

In fig. $3 \mathrm{a}$ and $4 \mathrm{a}$ clearances between the parts are shown with some exaggeration.

Using diamond pin, clearance in one direction $\left(c_{2}\right.$ in fig. $\left.3 \mathrm{a}\right)$ is much more than radial clearance, but contact surface area is smaller than round pin. Generally, the width of a diamond pin is usually $1 / 8$ of $\mathrm{D}[8]$.

The lateral clearance $c_{2 l}$ can be approximately calculated as [9]:

$$
c_{2 l} \approx c_{2 r} \frac{D_{2}}{b_{2}}
$$

A more accurate formula is [9]:

$$
c_{2 l}=\sqrt{b_{2}^{2}+D_{2}^{2}-d_{2}^{2}}-b_{2}
$$

The $2 \mathrm{H} 2 \mathrm{P}$ problem with two round pins was studied by A. Shan [10]. The probability of successful $2 \mathrm{H} 2 \mathrm{P}$ assemblies to specifications is estimated using a Monte Carlo simulation. Two inequations are used to calculate the feasibility of the $2 \mathrm{H} 2 \mathrm{P}$ assembly:

$$
D P I \geq D H I \text { and } D P O \leq D H O
$$

$D P I$ is inside distance between two pins; $D P O$ is outside distance between two pins; $D H I$ is inside distance between two holes; $D H O$ is outside distance between the holes.

In this paper, one round and one diamond locating pin are considered, the feasibility condition uses just one inequation (6). From this general condition the worst case and the assembly having two round pins result as particular cases.

During assembling the mounted module can be slightly moved, so the axis $\mathrm{O}_{12} \mathrm{O}_{22}$ and $\mathrm{O}_{11} \mathrm{O}_{21}$ are coincident. One critical position of two components has three contacts between pins and holes: $K_{1}, K_{2}$ and $K_{2}^{\prime}$ (fig. 3a). The assemblability criterion is:

$$
0.5\left(c_{1 r}+c_{2 l}\right) \geq L_{2}-L_{1}
$$

For the other critical position:

$$
0.5\left(c_{1 r}+c_{2 l}\right) \geq L_{1}-L_{2}
$$

From 4,5 and 2 inequations results the following assemblability criterion:

$$
0.5\left(c_{1 r}+c_{2 l}\right)=0.5\left(c_{1 r}+\sqrt{b_{2}^{2}+D_{2}^{2}-d_{2}^{2}}-b_{2}\right) \geq\left|L_{1}-L_{2}\right|
$$

If the both pins are round $b_{2}=d_{2}$ then $c_{2 l}=c_{2 r}$, from assemblability condition 6 results:

$$
0.5\left(c_{1 r}+c_{2 r}\right) \geq\left|L_{1}-L_{2}\right|
$$

A common source ofL $1, \mathrm{~L}_{3}$ errors are the position tolerances (TPp) of holes. Usually, the distributions are normal, the magnitude of the first vector-error $\varepsilon_{1}$ is $0 \ldots \mathrm{TPp} / 2$. Another parameter of the vector-error is the orientation. It is assumed that $\alpha_{l}$ is a random variable with a uniform distribution from 0 to $2 \pi$. The diamond head asymmetry must also be taken into consideration, when $\mathrm{L}_{2}$ is calculated:

$$
L_{2}=L_{3}+s \varepsilon_{s}, s=+1 \text { or }-1, \varepsilon_{s}=0 \ldots 0.5 T P_{s}
$$

If the worst case $(100 \%$ interchangeability) is considered, the following values are assigned to variables: minimum clearances, one of the centre distance has a minimum value 
and the other the maximum value, maximum width and asymmetry of the diamond pin, therefore the assemblability condition is:

$$
\begin{gathered}
0.5\left(c_{1 r m}+c_{2 l m}\right)=0.5\left(c_{1 r m}+\sqrt{b_{2 M}^{2}+D_{2 m}^{2}-d_{2 M}^{2}}-b_{2 M}\right) \geq T P p_{1}+T P p_{2}+0.5 T P s_{2} \\
0.5\left(c_{1 r m}+c_{2 l m}\right) \approx 0.5\left(c_{1 r m}+\frac{d_{2 m}}{b_{2 M}} c_{2 r m}\right) \geq T P p_{1}+T P p_{2}+0.5 T P s_{2}
\end{gathered}
$$

Usually $\mathrm{TPp}=\mathrm{TPp} \mathrm{p}_{1}=\mathrm{TPp}_{2}$ and $c_{r m}=c_{1 r m}=c_{2 r m}$, from condition 10 results:

$$
0.5 c_{m}\left(1+\frac{d_{2 m}}{b_{2 M}}\right) \geq 2 T P p+0.5 T P s_{2}
$$

If both sleeves are round:

$$
c_{m} \geq 2 T P p
$$

\subsection{Assemblability of $2 \mathrm{H} 2 \mathrm{P} 2 \mathrm{H}$}
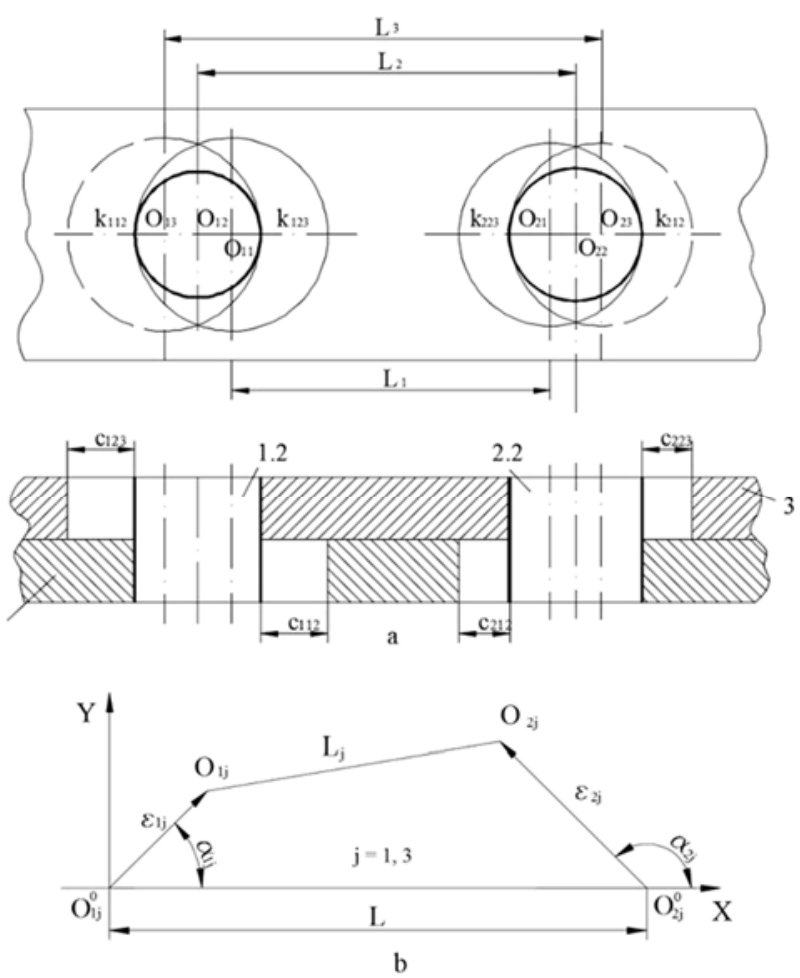

Fig. 4. a-2H2P2H in a limit assembly position; b-vectorial summation of centre distances.

In fig. $4 \mathrm{a}$ clearances are noted as: $\mathrm{c}_{\mathrm{ijk}}, \mathrm{i}=1,2 ; \mathrm{j}, \mathrm{k}=1-3, \mathrm{jk}$ defines de clearance between the assembly components, for example $c_{123}$ is clearance between sleeve/position screw 12 and mounted module 3 . Unlike the $2 \mathrm{H} 2 \mathrm{P}$ notations, because all clearances are radial, the $\mathrm{r}$ index is not used. 
The assemblability criterion for a general $2 \mathrm{H} 2 \mathrm{P} 2 \mathrm{H}$ assembly is:

$$
0.5\left(c_{112}+c_{123}+c_{212}+c_{223}\right) \geq\left|L_{1}-L_{3}\right|
$$

If the worst case is considered, similar to $2 \mathrm{H} 2 \mathrm{P}$, the following substitutions are made: minimum clearances $\left(c_{m}=c_{112}=c_{212}=c_{123}=c_{223}\right)$, one of the centre distance has a minimum value and the other the maximum value $\left(\left|L_{1}-L_{3}\right|=T P p_{1}+T P p_{2}=2 T P p\right)$, so assemblability criterion is:

$$
c_{m} \geq T P p
$$

\section{Statistical approach of MFSH assemblability problem using Monte Carlo simulation}

MCS is an efficient tool of tolerance analysis to the design of mechanical assemblies [1115]. It is applied as a pure statistical method, or in combination with other methods.

For $2 \mathrm{H} 2 \mathrm{P}$ and $2 \mathrm{H} 2 \mathrm{P} 2 \mathrm{H}$ assemblies the input variables are: position tolerance of grid pattern and mounted module, common nominal diameter of holes and sleeves, upper and lower specification limits of holes and pins, respectively. Particularly, for diamond pin the additional input variables are: the width of head and its tolerance, the symmetry tolerance. The successful 2H2P2H assemblies using Monte Carlo simulation can be calculated with following algorithm, which simulates the $2 \mathrm{D}$ assembly process of modules:

- After selecting the distributions of holes and pins diameters, the random values of $\mathrm{D}_{11}$, $D_{21}, d_{12}, d_{22}, D_{13}, D_{23}$ are generated and then the clearances are calculated: $c_{i 12}=D_{i 2}-d_{i 2}$, $\mathrm{c}_{\mathrm{i} 23}=\mathrm{D}_{\mathrm{i} 3}-\mathrm{d}_{\mathrm{i} 2}, \mathrm{i}=1,2$.

- The centre distances between axes of the two adjacent holes $\mathrm{L}_{\mathrm{i}}, \mathrm{i}=1,3$ are calculated (fig. $4 b)$. Random error-vector $\varepsilon_{i j}, \mathrm{i}=1,2, \mathrm{j}=1,3$, is generated $\varepsilon_{\mathrm{ij}}=0 \ldots \mathrm{TPp} \mathrm{p}_{\mathrm{j}} / 2$ where $\mathrm{TPp}_{\mathrm{j}}$ is the positional tolerance of the centering holes and $\alpha_{i j}$ is the direction of $\boldsymbol{\varepsilon}_{\boldsymbol{i} \boldsymbol{j}}$. The random variable $\alpha_{\mathrm{ij}}$ has a uniform distribution from 0 to $2 \pi$.

- If the assembly $2 \mathrm{H} 2 \mathrm{P} 2 \mathrm{H}$ is not feasible $0.5\left(c_{112}+c_{123}+c_{212}+c_{223}\right)<\left|L_{1}-L_{3}\right|$ it is indexed, then go to step 1.

- The calculating steps $1 \ldots .3$ are repeated with a large sample size, and the results are processed.

The algorithm for 2H2P follows similar steps, but clearances between locating module 3 and sleeves 2 have zero value and assemblability criterion is calculated with (11).

In the first step, this paper investigates the solutions to the assemblability problem offered by the MSFH designers (table 2).

In table 2, $q$ is the index of MSFH interchangeability. It is difference between the clearances and variation of the centre distances:

$$
\begin{aligned}
& q_{2 H 2 P}=c_{m}-2 T P p-0.5 T P s_{2} \\
& q_{2 H 2 P 2 H}=2\left(c_{m}-T P p\right)
\end{aligned}
$$

If $q \geq 0$ all assemblies are possible, therefore the Monte Carlo simulation is uselessly.

Monte Carlo simulations with a $10^{8}$ sample size show that in the majority of commercial MSFH the $100 \%$ interchangeability is not used (table 2).

The estimation of sensitivities is not a direct result of MCS. In the present work, the weight of minimum clearance, position tolerances of holes, pins and holes tolerances are analysed. The sensitivity information can be obtained using the method: holding the limits of the independent variables constant except one being studied. 
Table 2. Analysis of the commercial MFSH assemblability.

\begin{tabular}{|c|c|c|c|c|c|}
\hline \multirow[t]{2}{*}{ No. } & \multicolumn{2}{|l|}{ MFSH } & \multirow{2}{*}{$\begin{array}{c}q \\
{[\mu \mathrm{m}]}\end{array}$} & \multicolumn{2}{|c|}{ Distributions } \\
\hline & Name & Type & & normal & uniform \\
\hline 1 & AMF M12 [2] & \multirow{5}{*}{$2 \mathrm{H} 2 \mathrm{P} 2 \mathrm{H}$} & -10 & 1 & 0.99999998 \\
\hline 2 & AMF M16 [2] & & -10 & 1 & 1 \\
\hline 3,4 & Halder M12, M16 [3] & & -6 & 1 & 1 \\
\hline 5 & Kipp M8 [4] & & -20 & 1 & 0.99925001 \\
\hline 6 & Kipp M12,16 [4] & & 24 & 1 & 1 \\
\hline 7,8 & Norelem M6, M10 [5] & $2 \mathrm{H} 2 \mathrm{P}$ & -1 & 1 & 0.99994901 \\
\hline
\end{tabular}

Designers can improve the modular systems by calculating the weight that each variation contributes to resultant assembly variation.

The uniform distributions of the position tolerances of holes, pins and holes tolerances are considered. As is known the uniform distribution predicts greater variation than the normal distribution, but not as great as worst case.

Because few rejected assemblies per million will be predicted, in this research the sensitivity analysis uses $10^{9}$ sample size.

\subsection{Sensitivity analysis of $2 \mathrm{H} 2 \mathrm{P}$ assembly}

Sensitivity analysis uses a2H2P assembly with usual values as basis of comparison: basic size of holes and sleeves diameter $\mathrm{d}=12 \mathrm{~mm}$; pitch $\mathrm{p}=40 \mathrm{~mm}$; the positional tolerance of the centering holes $\mathrm{TPp}_{1}=\mathrm{TPp}_{2}=0.01 \mathrm{~mm}$; upper and lower limits for holes $\mathrm{UL}=0.01 \mathrm{~mm}$, $\mathrm{LL}=0 \mathrm{~mm}, \mathrm{~T}=\mathrm{UL}-\mathrm{LL}=0.01 \mathrm{~mm}$; upper and lower limits for pins $\mathrm{ul}=-0.01 \mathrm{~mm}, 1 \mathrm{l}=-0.02 \mathrm{~mm}$, $\mathrm{t}=\mathrm{ul}-1 \mathrm{l}=0.01 \mathrm{~mm}, \mathrm{c}_{\min }=0.01 \mathrm{~mm}$. The width of diamond sleeve is $10 \mathrm{~mm}$ with $0.01 \mathrm{~mm}$ symmetrical tolerance, diamond head asymmetry geometrical tolerance TPs $=0.01 \mathrm{~mm}$.

Results of Monte Carlo simulations using Mathcad are: 27/106 2H2P-RPRP (RP-round pin) and $87 / 10^{6}$ 2H2P-RPDP (DP-diamond pin)) assemblies are rejected, so high quality level can be considered.

Usually the $\mathrm{TPp}=0.01 \mathrm{~mm}$ (see table 1 ), holes with $\mathrm{TPp}<0.01 \mathrm{~mm}$ are too expensive. In this analysis $0.02 \mathrm{~mm}$ is the maximum value of positional tolerance.

Because minimum clearance is possible to be greater or smaller than $0.01 \mathrm{~mm}$, both variants are investigated: $\mathrm{c}_{\min }=0 \ldots 0.01 \mathrm{mmand} \mathrm{c}_{\min }=0.01 \ldots 0.02 \mathrm{~mm}$.

If $\mathrm{T}_{22}=[0.02 \ldots 0.4]$ rejects remain constant 87 per million, so designer can assign a general tolerance for linear dimensions, medium or coarse class (ISO2768-1), and the diamond pin can be cheaper.

In fig. 5 and 6 with a horizontal marker is shown 2700 rejects per million, corresponding to tree-sigma rule $( \pm 3 \sigma)$. Sensibility analysis of the $2 \mathrm{H} 2 \mathrm{P}$ assemblies reveals: - increasing of the centering holes positional tolerance (TPp) and decreasing of the minimum clearance $\left(\mathrm{c}_{\mathrm{min}}\right)$ have great influence on probability of successful $2 \mathrm{H} 2 \mathrm{P}$ assemblies comparatively with hole or pin tolerance ( $\mathrm{T}, \mathrm{t}$ ) (fig.5 and fig.6);

- if minimum clearance decreases results more rejected parts of the 2H2P-RPDP than 2H2P-RPRP assembly, as a result of diamond pin asymmetry;

- if minimum clearance increases a practically $100 \%$ probability is achieved ((fig.5 and fig.6);

- increasing or decreasing of diamond width tolerance has not effect on assemblability (fig.7);

- diamond pin asymmetry is more important than diamond width tolerance (fig.7), but the effect on assemblability is less important than positional tolerance of holes and minimum clearance; 
- increasing of hole or pin tolerance has a minor impact on assemblability (fig.5 and fig.7).

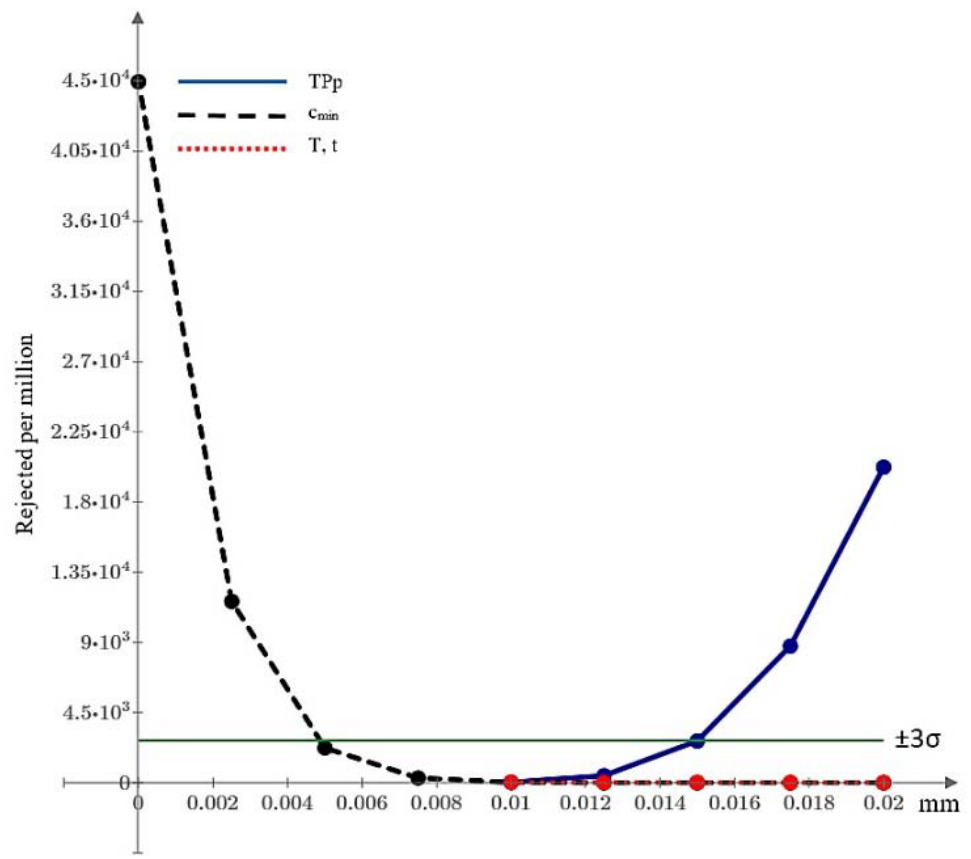

Fig. 5. Influence of positional tolerance of the centering holes, minimum clearance, hole or pin tolerance for 2H2P-RPRP.

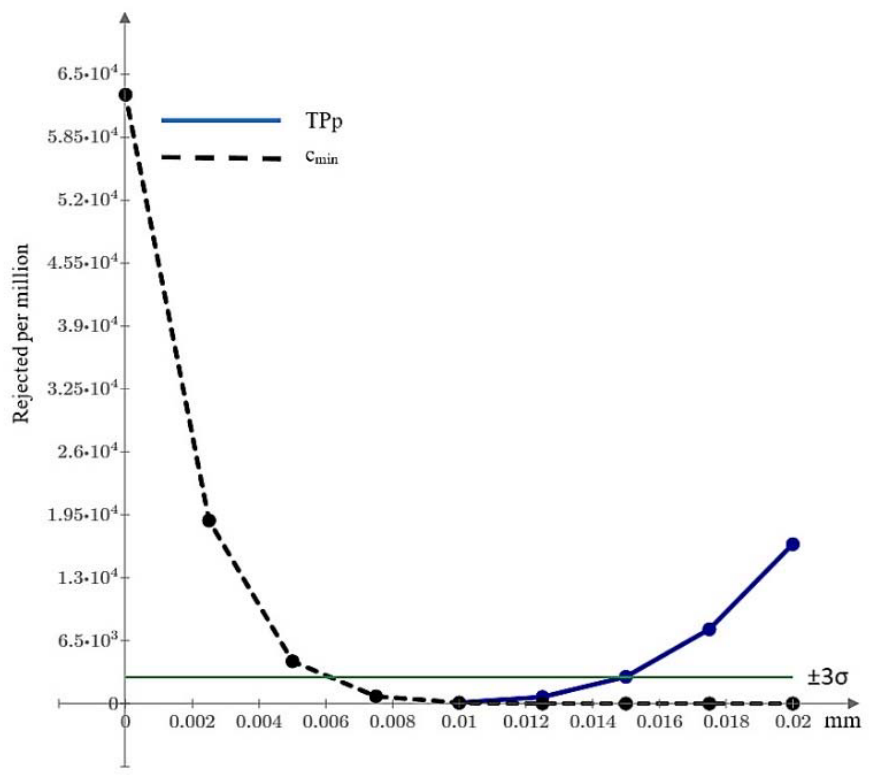

Fig. 6. Influence of positional tolerance of the centering holes and minimum clearance for $2 \mathrm{H} 2 \mathrm{P}-$ RPDP. 


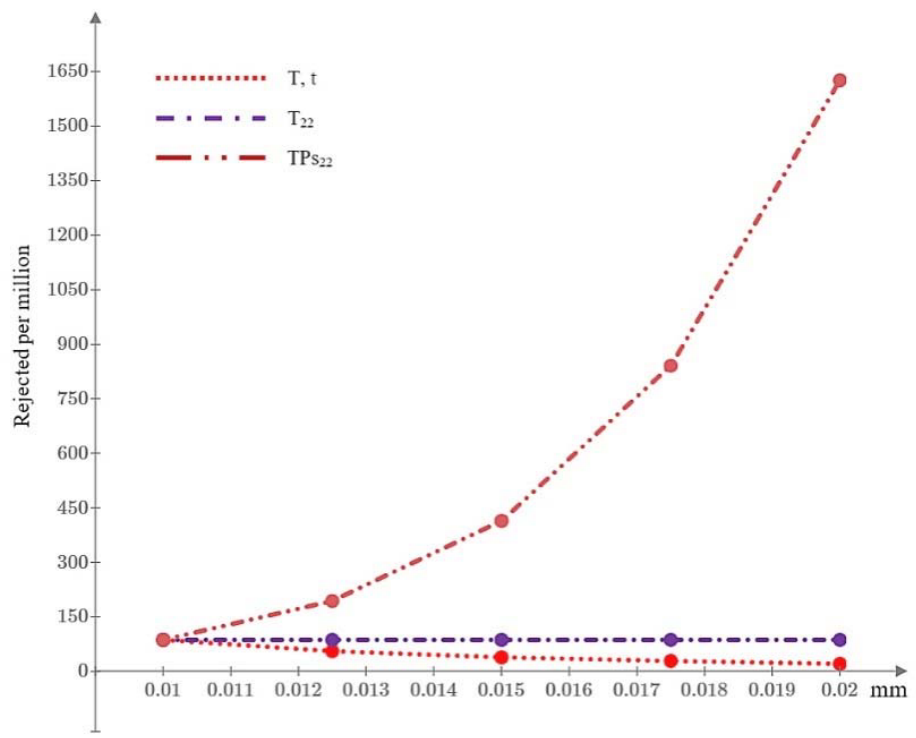

Fig. 7. Influence of hole or pin tolerance, the diamond pin width and symmetry tolerances for $2 \mathrm{H} 2 \mathrm{P}$ RPDP.

\subsection{Sensitivity analysis of $2 \mathrm{H} 2 \mathrm{P} 2 \mathrm{H}$ assembly}

The $2 \mathrm{H} 2 \mathrm{P} 2 \mathrm{H}$ assembly used as basis of comparison has the same values as $2 \mathrm{H} 2 \mathrm{P}-\mathrm{RPRP}$ assembly and the same sensitivity analysis method is applied.

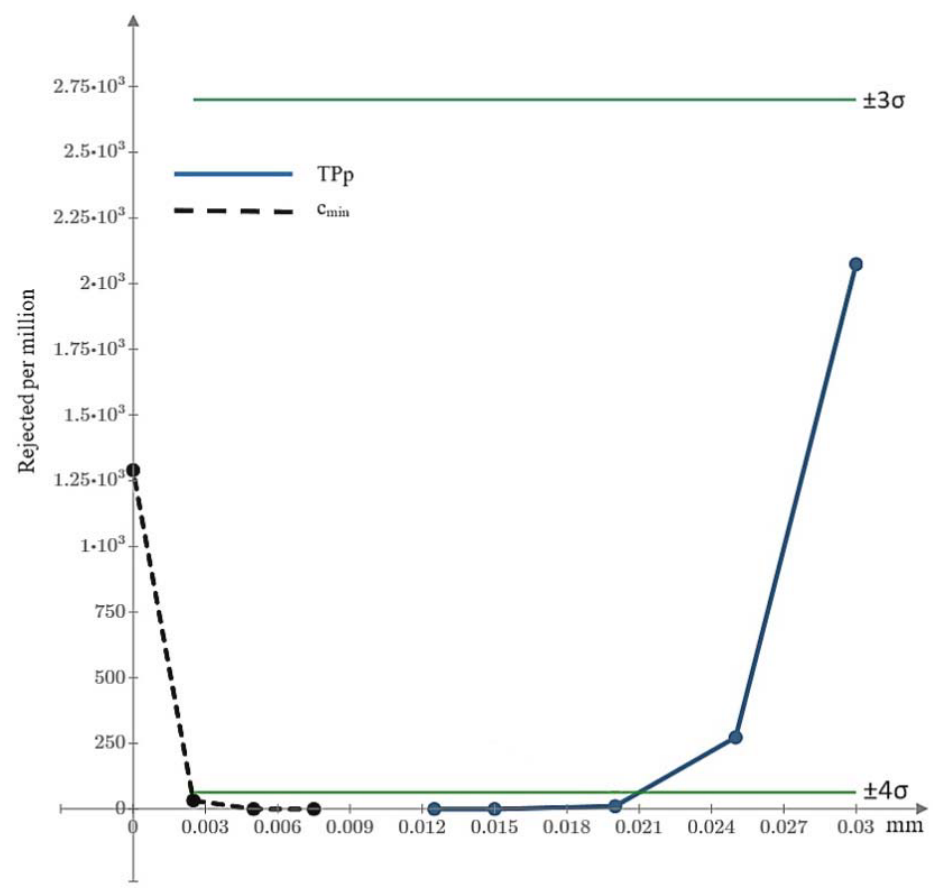

Fig. 8. Influence of the centering holes positional tolerance and minimum clearance for $2 \mathrm{H} 2 \mathrm{P} 2 \mathrm{H}$. 
Additionally, in fig. 8 and fig.9 with a horizontal marker is shown 63.4 rejects per million corresponding to four-sigma rule $( \pm 4 \sigma)$.

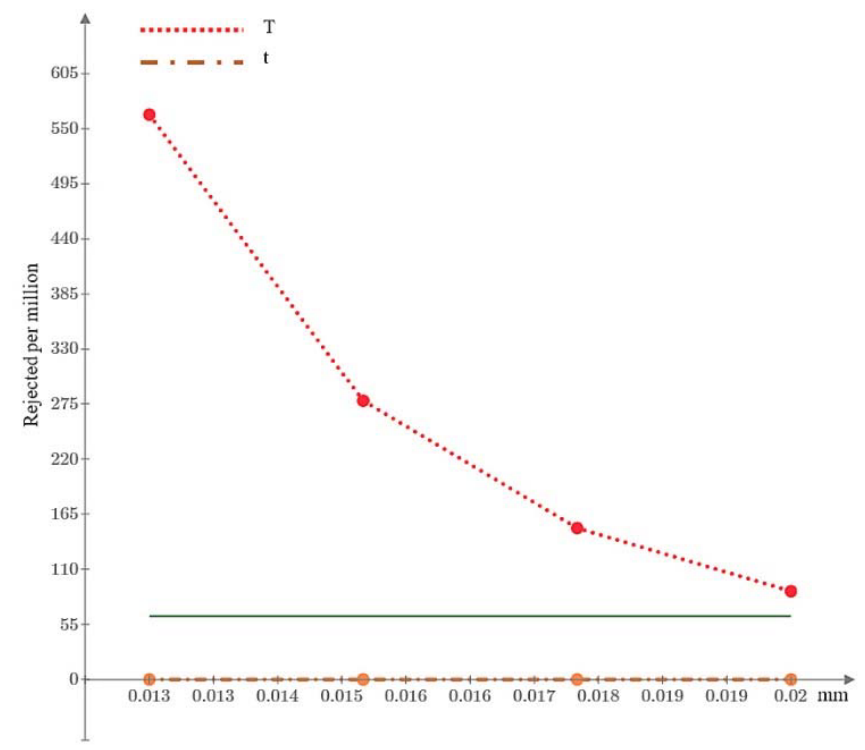

Fig. 9. Influence of hole and pin tolerance for $2 \mathrm{H} 2 \mathrm{P} 2 \mathrm{H}$.

Analysing the $2 \mathrm{H} 2 \mathrm{P} 2 \mathrm{H}$ dimensional sensibility reveals:

- if TPp $\in[0.0125 \ldots 0.02]$ hole positional tolerance increasing has minimum influence on probability of successful $2 \mathrm{H} 2 \mathrm{P} 2 \mathrm{P}$ assemblies, but if $T P p \in[0.02 \ldots 0.03]$ the influence is greater(fig. 8);

- influence of minimum clearances is greater if the locational clearance fit is considered $\left(\mathrm{c}_{\min }=0\right)$ (fig. 8);

- if hole tolerance is increased the number of rejected assemblies decreases (fig. 9);

- increasing of pin tolerance has zero impact on assemblability, because the worst case condition is satisfied (fig.9).

\section{Conclusions}

This paper focuses on the assemblability problem of the modular fixturing systems with accurate grid pattern holes in a statistical framework.

The two main purposes of this work are:

- to estimate the probability of successful $2 \mathrm{H} 2 \mathrm{P}$ and $2 \mathrm{H} 2 \mathrm{P} 2 \mathrm{H}$ assemblies;

- to analyse the sensitivity of these assemblies.

Stochastic models of the 2H2P-RPRP, 2H2P-RPDP and $2 \mathrm{H} 2 \mathrm{P} 2 \mathrm{H}$ assemblies are proposed. The assembly problem of modular fixturing systems with grid pattern holes is solved using Monte Carlo method.

The weights of minimum clearance, position tolerances of holes, pins and holes tolerances, tolerance and asymmetry of the diamond pin reveals:

- comparison of the results between the $2 \mathrm{H} 2 \mathrm{P}$ and $2 \mathrm{H} 2 \mathrm{P} 2 \mathrm{H}$ shows that the second assemblies can easier satisfy the assemblability condition;

- increasing of the centering holes positional tolerance and decreasing of the minimum clearance between pin and hole have great influence on probability of successful $2 \mathrm{H} 2 \mathrm{P}$ assemblies comparatively with hole or pin tolerance; 
- for 2H2P-RPDP assemblies, diamond pin asymmetry tolerance is more important than diamond width tolerance;

- hole or pin increasing tolerance has a minor impact on assemblability of $2 \mathrm{H} 2 \mathrm{P}$;

- if $\mathrm{TP}<0.02 \mathrm{~mm}$ hole positional tolerance increasing has minimum influence on probability of successful $2 \mathrm{H} 2 \mathrm{P} 2 \mathrm{P}$ assemblies, but if $\mathrm{TP}>0.02 \mathrm{~mm}$ the influence is greater;

- if hole tolerance is increased the number of rejected 2H2P2P assemblies decreases.

Based on this approach and results presented above, designers can optimize the modular fixturing systems with grid pattern holes for medium-size workpieces.

A future area of study is to integrate the results of the present study in an efficient method to optimize the fixturing modular systems correlated to the workpiece locating accuracy.

\section{References}

1. J. Marrs, Machine Designers Reference (Industrial Press Inc., New York, 2012)

2. N. P. Maniar, D. P. Vakharia, Int. J. of Scientific \& Eng. Research 4, 1 (2013)

3. Carr Lane modular fixturing systems, http://www.carrlane.com

4. Andreas Maier GmbH \& Co. KG, http://www.amf.de

5. Erwin Halder KG. http://www.halder.de

6. Heinrich Kipp Werk. http://www.kipp.com

7. Norelem. http://www.norelem.fr

8. P. H. Joshi, R. O. Parmley, Jigs and Fixtures Design Manual (McGraw-Hill Professional Publishing, New York, 2003)

9. S. Vasii-Rosculeţ, N. Gojinetchi, Proiectarea dispozitivelor [Fixture Design] (Didactic and Pedagogical Publishing., Bucharest,1982)

10. A. Shan, R. N. Roth, R. J. Wilson, Int. J. Adv. Manuf. Technol. 15, 222 (1999)

11. K. W. Chase, A. R. Parkinson, Research in Eng. Design 3, 23 (1991)

12. J. Gao, K. W. Chase, S. P. Magleby, Proc. of the ASME Design Eng. Tech. Conf., 1 (1995)

13. Y. S. Hong, T.C. Chang, Int. J. of Prod. Research 40, 2425 (2002)

14. F. Scholz, Boeing Information \& Support Services 1 (1995)

15. S. H. Huang, Int. J. Prod. Res. 42, 4871 (2004)

16. H. Geng, Manufacturing Engineering Handbook (McGraw-Hill Education Publishing, New York, 2016) 